\title{
Meninos e Meninas: Influências Culturais no Método de Zulliger
}

\author{
Lucila Moraes Cardoso ${ }^{1}$ \\ Universidade Estadual do Ceará, Fortaleza-CE, Brasil \\ Jamille Cavalcante de Oliveira \\ Hospital Universitário Walter Cantídio - Universidade Federal do Ceará, Fortaleza-CE, Brasil
}

\section{RESUMO}

Neste estudo, objetivou-se comparar o desempenho no Zulliger pelo Sistema Compreensivo (ZSC) em função do sexo biológico em crianças cearenses. Participaram do estudo 68 crianças, pareadas quanto ao sexo e a idade, variando entre 6 e 11 anos. Foram administrados nas crianças os testes de Raven e ZSC em sessão individual. O ZSC identificou nas meninas maior capacidade em demonstrar necessidades afetivas e um funcionamento cognitivo imaturo e, nos meninos, possível meticulosidade, tendências a se refugiar na fantasia, emprego distorcido da verbalização e dificuldades nos relacionamentos interpessoais. O fato do ZSC distinguir as disparidades observadas de acordo com gênero sugere evidências de validade para o método.

Palavras-chave: avaliação psicológica; validade do teste; teste de Zulliger; crianças.

\section{ABSTRACT - Boys and Girls: Cultural Influences in the Zulliger Method}

The objective of this study was to compare the performance in the Zulliger Comprehensive System (ZCS) as a function of biological sex in children from Ceará. The study included 68 children, divided equally in sex and age, ranging from 6 to 11 years. The Raven and ZCS tests were administered to the children individually. The ZCS identified girls' ability to demonstrate affective needs and immature cognitive functioning and, in boys, possible meticulousness, tendencies to take refuge in fantasy, distorted use of verbalization and difficulties in interpersonal relationships. The fact that the ZCS distinguishes observed gender disparities suggests evidence of validity for the method.

Keywords: psychological evaluation; test validity; Zulliger test; children.

\section{RESUMEN - Niños y Niñas: Influencias Culturales en el Método de Zulliger}

El objetivo fue comparar el rendimiento del test de Zulliger (ZSC) por el Sistema Comprensivo, en función del sexo biológico de niños cearenses. Participaron del estudio 68 niños, pareados por sexo y edad, variando la misma de 6 a 11 años. Se administraron los tests de Raven y ZSC en sesiones individuales. El ZSC identificó en las niñas mayor capacidad en demostrar necesidades afectivas y un funcionamiento cognitivo inmaduro, y, en los niños, posible meticulosidad, tendencia a refugiarse en fantasías, uso distorsionado de la verbalización y dificultades en las relaciones interpersonales. El hecho del ZSC distinguir las disparidades observadas de acuerdo con el género, sugiere evidencias de validez para el método.

Palabras claves: evaluación psicológica; validez del test de Zulliger; niños.

A avaliação psicológica é uma atividade ampla e complexa compreendida como um processo no qual há coleta de dados e interpretações de informações, por meio de teorias, métodos e instrumentos psicológicos, visando obter informações acerca de um indivíduo, um grupo ou situações específicas, e auxiliando em processos de tomada de decisões (Hutz, 2015). Dentre os procedimentos utilizados nos processos de avaliação psicológica, pode-se mencionar os métodos projetivos.

Os métodos projetivos podem ser utilizados na avaliação da dinâmica de personalidade ou de outros elementos, como relações interpessoais e dinâmica familiar, que se mostrem relevantes para a compreensão de um sujeito ou de uma situação por ele vivenciada. A estruturação pouco usual desses métodos, associada à tarefa proposta, permite conhecer o modo como o examinando percebe, estrutura e responde à situação estímulo, possibilitando o acesso a conteúdos que lhes são próprios e particulares (Fensterseifer \& Werlang, 2008).

Nas últimas décadas, esses métodos têm sido submetidos a rigorosos estudos estatísticos e qualitativos, 
o que fornece maior solidez para a interpretação de seus resultados (Miguel, 2014). Um dos requisitos mínimos que todo instrumento deve apresentar se refere às evidências de validade, essenciais na construção de um teste. Esse conceito faz alusão ao grau no qual as interpretações obtidas dos dados empíricos do teste encontram sustentação em base científica sólida e devem ser aferidas por meio de estudos que busquem verificar o quanto o instrumento realmente mede aquilo que se propõe a medir. Além disso, as evidências de validade de um teste mostram suas qualidades diante de um propósito ou de uma utilização particular, podendo um determinado instrumento servir a um objetivo de avaliação e não servir a outro diferente (Pacico \& Hutz, 2015).

Dentre os diversos métodos projetivos, o Zulliger pelo Sistema Compreensivo (ZSC) vem destacando-se pela quantidade de estudos desenvolvidos a seu respeito e pela qualidade destes. O ZSC fornece informações diversas sobre o examinando, incluindo aspectos como funcionamento cognitivo e afetivo, percepção e controle emocional.

O Zulliger foi criado em 1948, com base no método de Rorschach no que diz respeito às características do estímulo, aos procedimentos de classificação das respostas e de análise dos dados. Já o Sistema Compreensivo à análise e interpretação do Rorschach foi desenvolvido na década de 1970 (Exner \& Sendín, 1999), tendo o Rorschach pelo Sistema Compreensivo sido normatizado para o Brasil por Nascimento (2010).

Atualmente, O ZSC conta com uma amostra normativa de adultos tendo parecer favorável do Conselho Federal de Psicologia para uso, mas ainda não possui normas para a utilização em crianças. No Brasil, poucos são os métodos projetivos destinados à avaliação de crianças, o que tem feito alguns pesquisadores investirem na busca por evidências de validade e normas de tais métodos para esse público. Algumas dessas pesquisas indicam que o ZSC tem se mostrado útil na compreensão de aspectos da dinâmica de personalidade de crianças, como os estudos de evidências de validade de Villemor-Amaral e Quirino (2013), Tavella e Villemor-Amaral (2014), Biasi e Villemor-Amaral (2016), Villemor-Amaral, Pavan, Tavella, Cardoso e Biasi (2016) e Villemor-Amaral e Vieira (2016).

Na pesquisa de Villemor-Amaral e Quirino (2013), objetivou-se verificar se haveria correlação entre os tipos de resposta de cor no ZSC e o aspecto formal no teste das Pirâmides Coloridas de Pfister, considerando que ambos evidenciam os diferentes níveis de integração da percepção da cor com a forma. A amostra foi composta por 60 crianças, metade com 6 anos de idade e o restante com 12. Os resultados apontaram para a suposição de que, no Zulliger, as respostas de cor estão menos influenciadas pelo desenvolvimento cognitivo do que os indicadores de integração forma e cor no Pfister, contribuindo para o processo de validação de ambos os testes com o público infantil.

Tavella e Villemor-Amaral (2014) buscaram verificar se os indicadores cognitivos e afetivos no ZSC contribuíam para distinguir crianças com potenciais criativos diversos, identificadas pelo Teste de Criatividade Figural Infantil. Participaram do estudo 90 crianças, de 11 e 12 anos de idade. As crianças com níveis de criatividade alto apresentaram mais tendência a serem produtivas (R), com indícios de criatividade ( $\mathrm{M}, \mathrm{Ma}$ ) ao mesmo tempo que conseguem perceber elementos mais associados ao senso comum $(\mathrm{P})$, sugerindo que, por vezes, estavam mais propensas a uma visão excessivamente subjetiva, por vezes distorcida da realidade (FQ-). Concluindo-se, a partir disso, que as variáveis do Zulliger selecionadas foram sensíveis para identificar crianças com diferentes potenciais criatividade.

Biasi e Villemor-Amaral (2016), por sua vez, investigaram evidências de validade de critério para o ZSC, tendo como foco os indicadores de relacionamento interpessoal. Participaram do estudo 119 crianças, de ambos os sexos, do quarto ao sexto ano do ensino fundamental, que responderam a um sociograma para a seleção de dois grupos de participantes, formados por crianças consideradas mais ou menos populares. Os resultados demonstraram que indicadores do ZSC claramente associados na literatura com melhor qualidade nos relacionamentos interpessoais diferenciaram crianças mais populares e menos populares, sendo estes indicadores frequentemente considerados sinal de maior amadurecimento.

No estudo de Villemor-Amaral et al. (2016), comparou-se os desempenhos no ZSC de crianças em diferentes etapas do desenvolvimento. Participaram da pesquisa 103 crianças de 6 e 12 anos, tendo o ZSC diferenciado os participantes de modo coerente ao esperado teoricamente para suas idades, contribuindo com evidências de validade de uso do método com crianças.

Villemor-Amaral e Vieira (2016) buscaram por evidências de validade para uso do ZSC na avaliação da maturidade para o relacionamento interpessoal em crianças, por meio da comparação em função do sexo e idade cronológica. Participaram do estudo 115 crianças divididas em dois grupos com 6 e 12 anos. Após comparar o desempenho das crianças no ZSC, verificaram que os meninos apresentaram mais controle emocional (FC), com certa imaturidade intelectual e desconfiança (Ad). Já as meninas indicaram mais capacidade de empatia e de relacionamentos interpessoais $(\mathrm{H})$ com menor eficiência cognitiva (A). Além destes, é interessante ressaltar também a dissertação de mestrado de Carvalho (2015), em que a autora objetivou levantar dados normativos da população infanto-juvenil de Goiânia no ZSC, verificando possíveis influências acerca de sexo, origem escolar e idade. Participaram 304 crianças e adolescentes, de 7 a 14 anos, distribuídos em três grupos etários. Os resultados 
demonstraram diferenças significativas entre os grupos etários, os tipos de escolas e entre os sexos dos participantes, tendo sido estabelecidas normas para cada grupo etário e para os sexos de dois desses grupos.

A busca por evidências de validade dos instrumentos deve considerar as particularidades de cada contexto e situações às quais o teste poderá ser utilizado à tomada de decisão (Ambiel \& Carvalho, 2017), em especial, ao se considerar que o ser humano se desenvolve a partir de suas interações com ambiente. Como discutem Dornelles e Dal'Igna (2015), um indivíduo produz-se enquanto sujeito social pelos processos educativos diversos que são constituídos e legitimados em uma sociedade, sendo por meio destes conduzido para a realidade de uma determinada cultura.

No que diz respeito especificamente à influência cultural que é exercida sobre os sexos, que é o foco da presente pesquisa, Louro (2011) ressalta que a inscrição da masculinidade e da feminilidade nos corpos é feita, sempre, em um determinado contexto sociocultural, supondo uma construção e um aprendizado específicos de cada cultura. Nesse sentido, compreender as representações sociais relativas ao sexo biológico, e de que forma estas podem influenciar padrões de comportamento, afetividade e cognição, exige uma contextualização.

Vários pesquisadores se propõem a compreender como essas relações se desenrolam nos diversos âmbitos sociais. No que diz respeito à realidade brasileira e, especificamente, ao ambiente escolar, onde os aspectos culturais acerca do ser homem e ser mulher são também (re)construídos e (re)afirmados, destacam-se, como exemplo, os estudos de Dal'Igna (2007) e de Vianna e Finco (2009).

Dal'Igna (2007) analisou o modo como o sexo biológico é perpassado por construções sociais que incorporam, mobilizam, constituem e são constituídas pelo discurso pedagógico. A pesquisadora ouviu professoras de séries iniciais e pôde analisar como tais aspectos atravessam e dimensionam seus discursos. O comportamento dos alunos e suas dificuldades em disciplinas e conteúdos específicos foram relacionados, em alguns momentos, a aspectos de origem biológica e inata, o que evidenciou mecanismos de naturalização e dicotomização existentes no contexto estudado. Em um viés de investigação parecido e alcançando resultados semelhantes, a pesquisa de Vianna e Finco (2009) objetivou tratar das relações de gênero e poder presentes nos processos de socialização de crianças, também por meio da análise do relato de professoras. Por meio de dois casos citados pelas professoras, em que crianças transgrediram a fronteira de gênero que lhes era imposta, verificou-se como características naturalizadas e direcionadas à masculinidade e à feminilidade são, na verdade, resultantes da disciplina sobre comportamentos e corpos.

É importante destacar que tais discursos impostos sobre os sexos entrecruzam-se e relacionam-se com os demais discursos produtores de modos de relação e constituintes de identidades. Nesse sentido, pensar na realidade brasileira implica na necessidade de considerar as identidades regionais peculiares construídas na ampla extensão territorial do país, as quais são perpassadas também pelos discursos construídos acerca dos sexos.

Por meio da análise de músicas populares nordestina, Faria (2002) discute as noções de homem e mulher perpetradas na região Nordeste e refletidas nas letras de canções de dois expoentes do forró, estilo popular na região: o compositor e intérprete Luiz Gonzaga - década de 50 - e a banda Mastruz com Leite - década de 90. A autora expõe que, nessas décadas houve mudanças culturais, sociais e morais que exerceram sua força sobre as percepções disseminadas acerca de masculino e feminino.

Os contextos históricos e a realidade sociocultural em que se originaram as produções analisadas diferem, já que uma correnteza de mudanças advindas do próprio processo de pós-modernização atravessou o Nordeste enquanto região geográfica e enquanto região cultural, o que resultou em mudanças na condução das relações e nas produções de identidade. Os próprios padrões de comportamento dos indivíduos transformaram-se, emergindo padrões de sensibilidade novos que romperam com as imagens de masculinidade e feminilidade que habitavam até então o país e, especificamente, o Nordeste (Faria, 2002).

Tais aspectos imprimem, nos sujeitos, maneiras singulares de vivenciar suas relações de sexo e de gênero, de constituir suas identidades e de realizar suas escolhas. $\mathrm{Na}$ área de avaliação psicológica, em geral, não são consideradas as identidades de gênero, e sim o sexo biológico por ser uma variável mais concreta e mais fácil de ser observada (Villemor-Amaral \& Vieira, 2016).

Nesse sentido, objetiva-se com este trabalho buscar evidências de validade para uso do Zulliger pelo Sistema Compreensivo com crianças cearenses, por meio da comparação em função do sexo biológico. Justifica-se a importância do presente estudo no sentido de somá-lo às investigações que já estão sendo realizadas acerca do uso do método com crianças, ressaltando a necessidade de compreender se o instrumento é susceptível às influências culturais do meio em que os sujeitos estão inseridos, especificamente, às construções e elaborações relativas ao sexo e aos processos de subjetivação aí implicados.

\section{Método}

\section{Participantes}

A amostra foi composta por 68 crianças da capital cearense. As crianças foram pareadas em relação ao sexo e a idade, que variou entre 6 e 11 anos e 6 meses $(M=9,06$, $D P=1,39)$, conforme pode ser observada na Tabela 1 . Os critérios de inclusão na amostra foram cursar a série correspondente à sua faixa etária. Para controlar possíveis influências de fatores cognitivos, nesta pesquisa, foram selecionados somente os protocolos de crianças 
que tiveram um desempenho intelectual considerado médio inferior, médio e médio superior no teste das Matrizes Progressivas Coloridas de Raven. Além disso, constituíram-se como critérios de exclusão da amostra ter sido submetido, ou estar sendo submetido, a tratamento psicológico ou psiquiátrico.

Tabela 1

Distribuição da Idade, Tipo de Escola e Ano Escolar em Função do Sexo

\begin{tabular}{|c|c|c|c|c|c|c|c|}
\hline \multirow{2}{*}{\multicolumn{2}{|c|}{ Variável }} & \multicolumn{2}{|c|}{ Masculino } & \multicolumn{2}{|c|}{ Feminino } & \multicolumn{2}{|c|}{ Total } \\
\hline & & $f$ & $\%$ & $f$ & $\%$ & $f$ & $\%$ \\
\hline \multirow{6}{*}{ Idade } & 6 anos & 3 & 4,4 & 3 & 4,4 & 6 & 8,8 \\
\hline & 7 anos & 3 & 4,4 & 3 & 4,4 & 6 & 8,8 \\
\hline & 8 anos & 2 & 2,9 & 2 & 2,9 & 4 & 5,9 \\
\hline & 9 anos & 10 & 14,7 & 10 & 14,7 & 20 & 29,4 \\
\hline & 10 anos & 13 & 19,1 & 13 & 19,1 & 26 & 38,2 \\
\hline & 11anos & 3 & 4,4 & 3 & 4,4 & 6 & 8,8 \\
\hline \multirow{2}{*}{ Escola } & Pública & 16 & 23,5 & 19 & 27,9 & 35 & 51,5 \\
\hline & Particular & 18 & 26,5 & 15 & 22,1 & 33 & 48,5 \\
\hline \multirow{6}{*}{ Ano escolar } & $1^{\circ}$ & 4 & 5,9 & 3 & 4,4 & 7 & 10,3 \\
\hline & $2^{\circ}$ & 4 & 5,9 & 4 & 5,9 & 8 & 11,8 \\
\hline & $3^{\circ}$ & 5 & 7,4 & 3 & 4,4 & 8 & 11,8 \\
\hline & $4^{\circ}$ & 9 & 13,2 & 7 & 10,3 & 16 & 23,5 \\
\hline & $5^{\circ}$ & 12 & 17,6 & 16 & 23,5 & 28 & 41,2 \\
\hline & $6^{\circ}$ & 0 & 0,0 & 1 & 1,5 & 1 & 1,5 \\
\hline
\end{tabular}

\section{Instrumentos}

Matrizes Progressivas Coloridas de Raven (Angelini, Alves, Custódio, Duarte, \& Duarte, 1999). Esse teste permite a avaliação da inteligência, mais especificamente da inteligência fluída. Nesta pesquisa, foi usada a versão Colorida do Raven, com administração individual, e o tempo de aplicação foi de aproximadamente 15 minutos.

Nos estudos psicométricos do Raven, obteve-se coeficientes favoráveis para uso do instrumento. Foi utilizado o método das metades de Spearman-Brown entre os itens pares e ímpares para cada sexo, em cada faixa etária e, para a amostra total, obteve-se o coeficiente corrigido de 0,92 para o sexo masculino; 0,90 para o feminino e 0,92 para a amostra total. Além disso, houve aumento progressivo das médias em função da idade cronológica. Destaca-se que o instrumento atualmente possui parecer do CFP favorável para uso.

Teste de Zulliger pelo Sistema Compreensivo (Villemor-Amaral \& Primi, 2009). O ZSC é composto por três pranchas, com manchas de tinta ambíguas como estímulo. A aplicação do instrumento é individual e dura cerca de 30 minutos. Durante a fase da associação livre, as três pranchas são apresentadas ao examinando uma de cada vez numa sequência pré-determinada. Então, lhe é pedido que diga com que cada uma das manchas se parece, enquanto o examinador faz anotações literais da fala do examinando. Na segunda fase da administração, denominada inquérito, o examinador repete para o examinando as respostas dadas e solicita que ele aponte a área da mancha usada para dar a resposta, devendo dizer também quais características da mancha contribuíram com que ele visse o que relatou.

A codificação do instrumento é então realizada conforme a proposta do Sistema Compreensivo. Após a codificação das respostas, as frequências de todos os códigos são computadas para permitir interpretações com base em expectativas normativas. Tais cálculos e procedimentos são registrados no chamado Sumário Estrutural, que agrupa variáveis de acordo com áreas do funcionamento psíquico, o que favorece a análise psicométrica do teste (VillemorAmaral \& Primi, 2009; Exner \& Sendín, 1999).

\section{Procedimento}

Coleta de dados. Auxiliaram na coleta de dados oito graduandos do curso de Psicologia, que passaram por um treinamento para assegurar que seguissem as normas de administração e codificação dos instrumentos utilizados na pesquisa. Todos os protocolos codificados foram revisados e corrigidos pela professora, coordenadora da pesquisa.

Para facilitar o acesso às crianças, foram contatadas duas escolas públicas e duas particulares, que disponibilizaram salas para que os testes fossem aplicados de forma individual nas próprias escolas em espaços, dias e horários previamente combinados. Além disso, enviaram os TCLEs aos responsáveis pelas crianças. Cada criança respondeu primeiro ao teste das Matrizes Progressivas 
Coloridas de Raven e, em seguida, o Zulliger, em sessão única e individual de aproximadamente 40 minutos.

\section{Análise dos Dados}

As informações descritivas da amostra e o desempenho das crianças em cada um dos testes foram inseridas em um banco de dados e exportados para um software de análises estatísticas. Foi realizada a estatística descritiva das características sociodemográficas dos examinandos, análise kappa de confiabilidade entre juízes com $25 \%$ dos protocolos para minimizar a probabilidade de erros decorrentes de codificação inconsistente; e o $t$ de Student, visando a comparação da média no desempenho das variáveis que compõe o Sumário Estrutural do ZSC em função do sexo. Além disso, foi calculado o $d$ de Cohen para analisar o efeito da magnitude (Cohen, 1992).

\section{Considerações Éticas}

Destaca-se que este estudo é parte de uma pesquisa intitulada "Evidências de Validade e Normatização de Métodos Projetivos para Crianças do Nordeste Brasileiro". A pesquisa foi aprovada no Comitê de Ética em Pesquisa (CEP/CONEP), sob parecer no 618.913.

\section{Resultados}

Das 90 variáveis do ZSC analisadas, 27 não tiveram índice de precisão aferidos por não aparecerem nos $25 \%$ de protocolos sorteados [map, C', YF, FV, VF, V, T, FD, Fr, rF, Cn, FQ+, FQnone, (Hd), (Ad), Cl, Ls, Ge, Xy, Ex, DV2, DR, DR2, INC2, FAB2, AB, MOR]. Além disso, o segundo juiz, erroneamente, deixou de codificar os códigos especiais GHR e PHR. Das variáveis que tiveram o coeficiente kappa analisado, 22 tiveram valores excelentes, acima de 0,81 , sendo elas W, D, Dd, DQ+, Ma, FMa, C, FC', F, FQo, Par, H, (H), Hd, A, Ad, Art, Fi, P, CON, AG, PSV e Bt e 17 foram consideradas boas, com coeficiente kappa entre 0,61 e 0,80, a saber, S, DQo, DQv, FC, CF, C'F, FY, FQu, FQ-, Na, Food, Bl, Cg, Hh, Sc, Id, DV. Além disso, quatro foram consideradas moderadas, com valores entre 0,41 e 0,60, sendo elas Mp, Ay, An e PER e duas com valores entre 0,21 e 0,40, INC e COP. As outras quinze variáveis não tiveram um nível de significância aceitável (FMp, Sx, Hx, CP, FMap, ma, mp, Y, FT, TF, (A), FAB, ALOG, DQv, Map). A partir desses resultados, os dois juízes identificaram os pontos de inconsistência nas variáveis e entraram em consenso sobre qual seria a codificação correta. Posteriormente, revisaram todos os protocolos do estudo, corrigindo possíveis equívocos.

Após adequação do banco, foi feita a comparação de média das variáveis do sumário estrutural do ZSC entre os sexos e verificou-se que 12 apresentaram diferenças estatisticamente significativas entre meninos e meninas, e a variável FT que foi marginalmente significativa. É importante frisar que o efeito de magnitude de todas essas diferenças variou de moderado até forte, com destaque às variáveis PHR e M- com os maiores efeitos de magnitude.

As variáveis que apresentaram diferenças significativas de acordo com o sexo das crianças podem ser encontradas na Tabela 2, sendo expostas conforme sequência do Sumário Estrutural do Zulliger pelo Sistema Compreensivo. Duas variáveis tiveram frequência aumentada no grupo das meninas, e onze mostraram-se elevadas no grupo dos meninos.

Tabela 2

Variáveis do ZSC que Tiveram Diferenças Significativas em Função do Sexo

\begin{tabular}{|c|c|c|c|c|c|c|c|}
\hline Variável & Sexo & $N$ & $M$ & $D P$ & $t$ & $p$ & $d$ \\
\hline \multirow{2}{*}{$\mathrm{Dd}$} & Feminino & 34 & 0,82 & 1,193 & \multirow{2}{*}{$-2,029$} & \multirow{2}{*}{0,047} & \multirow{2}{*}{$-0,5$} \\
\hline & Masculino & 34 & 1,56 & 1,744 & & & \\
\hline \multirow{2}{*}{ DQv } & Feminino & 34 & 0,71 & 1,142 & \multirow{2}{*}{2,203} & \multirow{2}{*}{0,033} & \multirow{2}{*}{0,57} \\
\hline & Masculino & 34 & 0,24 & 0,496 & & & \\
\hline \multirow{2}{*}{$\mathrm{Mp}$} & Feminino & 34 & 0,09 & 0,288 & \multirow{2}{*}{$-2,259$} & \multirow{2}{*}{0,028} & \multirow{2}{*}{$-0,56$} \\
\hline & Masculino & 34 & 0,32 & 0,535 & & & \\
\hline \multirow{2}{*}{$\mathrm{FT}$} & Feminino & 34 & 0,18 & 0,521 & \multirow{2}{*}{1,977} & \multirow{2}{*}{0,056} & \multirow{2}{*}{0,69} \\
\hline & Masculino & 34 & 0 & 0 & & & \\
\hline \multirow{2}{*}{ An } & Feminino & 34 & 0,21 & 0,479 & \multirow{2}{*}{$-2,047$} & \multirow{2}{*}{0,046} & \multirow{2}{*}{$-0,51$} \\
\hline & Masculino & 34 & 0,53 & 0,788 & & & \\
\hline \multirow{2}{*}{ DV } & Feminino & 34 & 0,53 & 0,748 & \multirow{2}{*}{$-2,027$} & \multirow{2}{*}{0,047} & \multirow{2}{*}{$-0,5$} \\
\hline & Masculino & 34 & 1 & 1,128 & & & \\
\hline \multirow{2}{*}{ PHR } & Feminino & 34 & 0,71 & 0,676 & \multirow{2}{*}{$-3,375$} & \multirow{2}{*}{0,001} & \multirow{2}{*}{$-0,82$} \\
\hline & Masculino & 34 & 1,38 & 0,954 & & & \\
\hline
\end{tabular}


Tabela 2 (continuação)

Variáveis do ZSC que Tiveram Diferenças Significativas em Função do Sexo

\begin{tabular}{cccccccc}
\hline Variável & Sexo & $N$ & $M$ & $D P$ & $t$ & $p$ & $d$ \\
\hline \multirow{2}{*}{ Blends } & Feminino & 34 & 0,26 & 0,511 & $-2,078$ & 0,043 & $-0,54$ \\
& Masculino & 34 & 0,68 & 1,036 & & & \\
M_ & Feminino & 34 & 0 & 0 & & & $-0,93$ \\
& Masculino & 34 & 0,18 & 0,387 & $-2,659$ & 0,012 & \\
FM & Feminino & 34 & 0,59 & 0,657 & & & $-0,54$ \\
& Masculino & 34 & 1 & 0,853 & $-2,231$ & 0,029 & \\
FM+m & Feminino & 34 & 0,62 & 0,652 & & & \\
& Masculino & 34 & 1,12 & 0,913 & $-2,598$ & 0,012 & $-0,64$ \\
Sum6 & Feminino & 34 & 0,59 & 0,783 & & & \\
& Masculino & 34 & 1,21 & 1,25 & $-2,442$ & 0,018 & $-0,61$ \\
WSum6 & Feminino & 34 & 0,65 & 0,884 & & & \\
& Masculino & 34 & 1,97 & 2,634 & $-2,778$ & 0,008 & $-0,75$ \\
\hline
\end{tabular}

\section{Discussão}

Inicialmente serão discutidos os indicadores aumentados nas meninas (FT e DQv). Conforme observado na Tabela 2, a variável FT foi marginalmente significativa com moderado efeito de magnitude $(p=0,56 ; d=0,69)$ e a variável DQv foi significativa também com efeito moderado $(p=0,033 ; d=0,57)$. As variáveis aumentadas nas meninas indicam maior capacidade para demonstrar necessidades afetivas (FT) e um processamento cognitivo mais primitivo e imaturo (DQv), conforme apontado por Exner e Sendin (1999). Essa necessidade de contato afetivo nas meninas (FT) faz sentido ao pensar na discussão que Vianna e Finco (2009) fazem ao tratar dos processos de educação dos corpos desde cedo ensinado às crianças, de modo que demonstrações de afeto e carinho são frequentemente incentivadas nas meninas, enquanto nos meninos tais comportamentos são muitas vezes cerceados. Isso se torna perceptível na maneira como a família ou os professores elogiam a meiguice das meninas, seu jeito carinhoso de ser. Mecanismos de naturalização e dicotomização aparecem então nos discursos de pais e educadores, como ressalta Dal'Igna (2007), na tentativa de justificar essa diferença entre meninos e meninas.

São eleitos gestos, comportamentos e posturas considerados adequados para rapazes e para moças, enquanto aquilo que estaria fora da norma é desconsiderado. Nesse sentido, Louro (2011) afirma que as práticas afetivas entre meninas e mulheres parecem ter um leque de expressões mais amplo do que aquele admitido para garotos e homens, incluindo uma intimidade por vezes demonstrada nos toques físicos ou na expressão das emoções, comportamentos dificilmente incentivados entre os homens.

Da mesmo forma, os indicadores referentes a uma forma de processamento mais primitiva e imatura (DQv) mais frequente nas meninas dessa amostra corrobora com a distinção sutilmente colocada à forma como se lida com as dificuldades de aprendizagem enfrentadas por meninos e meninas. De acordo com Dal'Igna (2007), ao encaminhar os meninos às aulas de reforço, as professoras costumam adotar adjetivos como preguiçoso, desatento, relaxado ou desinteressado, enquanto às meninas, ao contrário, mencionam apenas dificuldade e não eram necessárias explicações dessa dificuldade, ela simplesmente existia, naturalmente. A partir disso, Dal'Igna (2007) discorre que o desempenho abaixo da média de conhecimentos esperados de meninos e meninas são classificados e hierarquizados de formas diferentes. Meninos não atingiriam a média devido ao seu comportamento, enquanto meninas teriam suas dificuldades justificadas por sua capacidade cognitiva inferior, não atingindo a média por sua pura falta de conhecimento.

No estudo de Carvalho (2015), o processamento primitivo (DQv) esteve aumentado em meninos entre 7 e 9 anos e não encontrou diferenças por sexo nas demais idades. Na presente pesquisa, o controle da variável idade foi feito pareando as amostras, mas devido a quantidade pequena de crianças em cada faixa etária, não foi possível a comparação para cada uma das idades, o que sem dúvidas seria interessante de ser melhor investigado em estudos futuros. Além disso, de acordo com Exner e Sendín (1990), seria comum crianças pequenas apresentarem DQv, diminuindo a frequência desse indicador conforme a idade aumenta, principalmente a partir dos dez anos.

As demais variáveis presentes na Tabela 2 apresentaram maior frequência no grupo dos meninos. $\mathrm{O}$ indicador Dd estaria relacionado a um processamento mais minucioso, meticuloso e metódico das informações, uma busca de exatidão e uma percepção do que é menos 
óbvio (Villemor-Amaral \& Primi, 2009). De acordo com Nascimento (2010), refletiria uma tendência a observar o incomum, indicando também um aumento da meticulosidade e do perfeccionismo quando sobressalente em um protocolo. Ademais, poderia decorrer de uma aproximação muito pessoal ao campo de estímulos ou, ainda, funcionar como forma de evitar confrontações frontais com esse campo de estímulos (Exner \& Sendín, 1999).

É interessante observar que maior atenção aos detalhes é uma característica atribuída, no senso comum, às meninas. Em seus estudos, Dal'Igna (2007) e Vianna e Finco (2009) se depararam com o discurso de docentes que afirmaram serem as meninas naturalmente mais caprichosas, atenciosas, cuidadosas e preocupadas com os detalhes. No entanto, na aplicação do método, os meninos se atentaram mais aos detalhes que as meninas, reforçando a ideia de Louro (2011) de que os sujeitos não são receptores passivos das imposições externas. Eles estão envolvidos ativamente neste processo - reagindo, respondendo, recusando ou assumindo inteiramente o que lhes é colocado.

Indicativos de ideações deliberadas e tendências em se refugiar na fantasia (Mp) também estiveram aumentadas no grupo dos meninos. Como aponta Exner e Sendín (1999), a presença desse indicador envolve uma criação pessoal do examinando, incluindo tendências em compensar frustrações da realidade no campo da fantasia. As tentativas de interpretar tal indicador de modo isolado seriam imprudentes devido à complexidade de atividades com as quais essa variável se relaciona. As ideações deliberadas foi uma característica encontrada também com maior frequência nos meninos pelo estudo de Carvalho (2015).

Destacou-se também, no grupo dos meninos, um possível aumento da preocupação com o corpo (An), fornecendo indícios de alterações da autoimagem e das atitudes em relação a si mesmo. No estudo realizado por Carvalho (2015), preocupações corporais também se ressaltaram nos meninos, representadas pelo conteúdo Bl. Exner e Sendín (1999) ressaltam a importância de se estar atento aos momentos em que o corpo sofre alterações, pois estar vivenciando algum desses períodos pode ser o motivo de uma maior preocupação com o corpo e com a autoimagem.

Nesse sentido, pode-se pensar na infância também como uma etapa de mudanças no corpo, principalmente nas faixas etárias mais próximas da adolescência. $76,4 \%$ da amostra que integrou a pesquisa é composta por crianças com idade entre 9 e 11 anos, já mais próximas do período em que tais transformações no corpo se acentuam. Quanto ao fato desse código aparecer com mais frequência no grupo dos meninos, há a possibilidade do corpo ou da imagem corporal estar de alguma forma sendo foco para esses garotos. Faria (2002) ressalta que a preocupação com o corpo, que deve seguir padrões específicos para ser considerado socialmente belo e desejável, passou a ser um aspecto cobrado no Nordeste, não apenas ao feminino, como ocorreu ao longo de décadas, mas também ao masculino. Essa atenção excessiva à imagem corporal, que perpassa a constituição do ser feminino e ser masculino, responderia à um projeto da sociedade atual de consumo, que divulga imagens pré-determinadas de ser e viver, e vende supostas formas de se alcançar tais objetivos.

Mostrou-se mais frequente no grupo dos meninos indicativos de um uso distorcido da linguagem (DV), que tem relação com a utilização de palavras incorretas ou inadequadas, como, por exemplo, neologismos e redundâncias (Nascimento, 2010). Pode implicar breves lapsos de pensamento, decorrentes do emprego distorcido da verbalização, diminuindo a clareza e a precisão na comunicação do indivíduo. Pode também assinalar uma forma mais grave de deslize cognitivo, que pressupõe um uso de linguagem sem valor comunicativo (Exner \& Sendín, 1999).

É necessário, porém, ter cautela com a linguagem infantil e com as gírias. Como aponta Exner e Sendín (1999), estas podem ser interpretadas como neologismos sem que assim o sejam. Além disso, destaca-se a ressalva feita por Nascimento (2010) de que a presença desse indicador pode ser reflexo do mau uso da linguagem, comum na população brasileira. O Nordeste, assim como as demais regiões do país, possui peculiaridades linguísticas próprias de sua cultura, que podem soar incompreensíveis aos ouvidos daqueles que desconhecem seus significados. O uso de gírias foi identificado em muitas das respostas dadas por meninos e meninas, além do uso inadequado de determinadas palavras, já que os participantes desta pesquisa ainda estão em processo de aprendizado da linguagem formal.

Além disso, o fato dos meninos terem apresentado o indicador com maior frequência pode ter relação com a forma como habilidades distintas são incentivadas para meninos e meninas. De acordo com Louro (2011), a escola se constituiu, historicamente, como um campo normalizador e disciplinador, servindo-se de símbolos e códigos para afirmar o que cada um pode ou não fazer. A vida escolar seria permeada, portanto, por processos educativos escolares e não escolares, como ressaltam Dornelles e Dal'Igna (2015).

Nessa linha de raciocínio, ressalta-se que meninos e meninas desenvolveriam então certas habilidades e outras não. No estudo de Dal'Igna (2007), aparece no discurso das professoras entrevistadas a questão da habilidade verbal como sendo uma característica forte nas meninas, o que talvez mantenha relação com o fato do grupo de meninas ter apresentado o indicador com menos frequência. De acordo com as docentes, as meninas demonstrariam desde cedo uma facilidade maior para esse campo do conhecimento, sendo mais perceptivas e observadoras, além de conseguirem manter a concentração por mais tempo. Já os rapazes teriam mais facilidade 
em campos do conhecimento caracterizados pelo predomínio do pensamento abstrato, sendo suas habilidades voltadas, principalmente, para as atividades práticas.

Como é possível visualizar na Tabela 2, foi mais frequente nos meninos a presença de indícios referentes a dificuldades e conflitos na esfera dos relacionamentos interpessoais (PHR), refletindo possíveis fracassos nas relações e tendências a comportamentos sociais inadequados. No estudo de Villemor-Amaral e Vieira (2016), embora o aumento do PHR nos meninos tenha tido valores que não foram considerados estatisticamente significativos, outros indicadores também sugeriram maior desconfiança nas relações interpessoais.

Faria (2002), em sua reflexão acerca da constituição das identidades de gênero no Nordeste, ressalta a importância dada, no tradicionalismo nordestino, ao homem valente, "cabra macho", rústico e áspero. A construção inicial da figura do nordestino estava imbuída do sentimento de preservação da região, caracterizando-se culturalmente em defesa do patriarcalismo e do tradicionalismo, e opondo-se ao moderno e suas superficialidades. Havia, conforme descrição de Farias (2002), um realce das próprias características naturais da região, assim apenas um "homem de verdade", forte e viril, sobreviveria à natureza árida, ressequida e agressiva do sertão nordestino.

Os meninos também apresentaram mais indícios de uma capacidade cognitiva e flexibilidade para lidar com as demandas cotidianas (Determinantes Mistos - blends). Esse dado corrobora parte dos resultados encontrados por Carvalho (2015).

Exner e Sendín (1999) e Nascimento (2010) afirmaram que a presença desse indicador só será benéfica se a pessoa dispor de abundantes recursos, o que aumentaria suas possibilidades de respostas diante de situações diversas. Já se os recursos forem escassos, colocará em risco a estabilidade e a coerência interna do indivíduo. Desse modo, a análise desse indicador demandaria associação com os demais elementos presentes nos protocolos de cada uma das crianças que tiveram aumento de blends, sendo contraindicada a análise dessa variável de modo isolado.

Indicativos de certo grau de desorientação do pensamento e afastamento da realidade mostraram-se mais altos no grupo dos meninos (M-), porém, como ressalta Nascimento (2010), tal indicador terá significação mais importante se aparecer várias vezes em um único protocolo. Como visto na Tabela 2, a presença de $\mathrm{M}$ - foi nula no grupo das meninas e muito baixa no grupo dos meninos. Embora a diferença dessa variável tenha sido significativa $(p=0,012)$ e de elevada magnitude de efeito $(d=$ $0,93)$, defende-se que esse dado deva ser considerado com cautela, pois o fato dos meninos terem produzido mais respostas M- não necessariamente indicaria psicopatologia na medida em que a frequência foi muito baixa.

Dois indicadores com significações semelhantes mostraram-se relevantes no grupo dos meninos, apontando para indícios de uma atividade mental não deliberada e não controlada (FM) e de pensamentos que fazem pressão e invadem a mente do indivíduo, sem que ele tenha condições de deter ou controlar esse processo $(F M+m)$. De acordo com Exner \& Sendín (1999) e Nascimento (2010), tais ideações não deliberadas seriam provocadas por estados de insatisfação das necessidades básicas, tanto primárias (fome, sede, etc.) quanto secundárias (sucesso, prestígio, etc.), podendo contribuir para uma sobrecarga interna. Além disso, seriam mais frequentes em protocolos de crianças.

Possíveis dificuldades de pensamento (Sum6 e Wsum6) foram mais frequentes nos protocolos dos meninos. Exner e Sendín (1999) enfatizaram que tais indicadores apontam para um possível prejuízo na capacidade de pensar de forma lógica e coerente. Essas variáveis apresentam níveis de gravidade distintos, podendo indicar desde pequenos deslizes no pensamento até comprometimentos ideacionais severos. Analisando os protocolos dos meninos, verificou-se que o aumento desse código decorre da frequência elevada do código especial DV, que é o indicador de menor peso à atribuição de dificuldades psicopatológicas mais severas e já foi discutido no presente artigo.

Outro ponto a ser ressaltado diz respeito ao fato dos códigos Mp, M-, Sum6 e WSum6, todos mais frequentes nas respostas dadas pelos meninos, fazerem parte do módulo de Ideação, presente no sumário estrutural. As variáveis desse agrupamento mostram como o indivíduo conceitualiza a informação que foi colhida e traduzida pelos outros dois processos, de Mediação e de Processamento da Informação. De acordo com Nascimento (2010), no módulo de Ideação é analisada a predominância ou não dos processos de pensamento sobre as emoções e a sua qualidade. Em outras palavras, essa sessão traz indicativos sobre como a pessoa pensa a respeito daquilo que vivencia e como pensa sobre essas experiências ao tomar suas decisões.

Nesse sentido, nota-se que os códigos referentes ao módulo de Ideação nas respostas fornecidas pelos meninos dessa amostra, atrelada aos sentidos possíveis atribuídos a esses indicadores quando presentes em um protocolo, aponta para especificidades do grupo no âmbito do pensamento. De acordo com Nascimento (2010), depois de apreender as informações da realidade e traduzi-las, o indivíduo reflete a respeito dessas informações e toma decisões diante disso. Possivelmente esse processo se dá de forma mais fantasiosa e distante da realidade no grupo dos meninos do que das meninas dessa amostra, como mostram os resultados deste estudo e as discussões aqui tecidas. Além disso, ressalta-se que tal refúgio na fantasia e afastamento da realidade pode constituir-se de diferentes formas e em diversos níveis, necessitando cautela ao se lidar com esses dados.

Destaca-se que o conjunto dos indicadores que tiveram diferenças significativas são indícios percebidos pelo uso ZSC e que indicam peculiaridades no 
funcionamento psíquico dos grupos estudados. As diferenças na média dos indicadores ora apresentados e seus significados demonstram, portanto, que o método de Zulliger identificou particularidades do funcionamento psíquico dos meninos e das meninas que compuseram a amostra desta pesquisa. As diferenças entre os dois grupos estão em consonância com questões culturais e históricas relacionadas a percepção do papel masculino e feminino ainda presentes no modo de ser das crianças da amostra estudada.

Os resultados do presente estudo, assim como os de comparação do Zulliger por sexo com crianças de Goiânia - GO (Carvalho, 2015) e do estado de São Paulo e Minas Gerais (Villemor-Amaral \& Vieira, 2016) sugerem que o ZSC consegue identificar peculiaridades do funcionamento de meninas e meninos. Destaca-se que, embora as variáveis que evidenciaram as diferenças não tenham sido as mesmas, o presente estudo corrobora achados obtidos no estudo de Villemor-Amaral e Vieira (2016) na medida em que, nos dois estudos, os meninos evidenciaram maior inclinação do que as meninas para aspectos concretos e lógicos e também maior desconfiança nas relações interpessoais.

É importante ressaltar que o tamanho da amostra utilizada é bastante limitado, impossibilitando que o resultado desta pesquisa seja generalizado ao tratar de crianças do Ceará. Além disso, ainda que se tenha tentado controlar o efeito da idade equiparando os dois grupos (Tabela 1), há uma variedade nas idades que pode ser controlada em estudos futuros.

Ressalta-se a importância de continuidade dos estudos para uso do ZSC no Ceará, bem como de ampliar os estudos com o Sistema Compreensivo e de serem realizadas pesquisas com amostras maiores e em distintos contextos socioculturais, tal como foi defendido por VillemorAmaral e Quirino (2013), Tavella e Villemor-Amaral (2014), Carvalho (2015), Biasi e Villemor-Amaral (2016), Villemor-Amaral et al. (2016) e Villemor-Amaral e Vieira (2016). Tal movimento é essencial para que se continue a tarefa de contribuição às evidências de validade do método e ampliação do leque de situações, contextos e públicos em que é possível um uso adequado do instrumento.

\section{Referências}

Ambiel, R. A.M., \& Carvalho, L. F. (2017). Validade e precisão de instrumentos de avaliação psicológica. Em M. R. C. Lins \& J. C. Borsa (Eds.). Avaliação Psicológica: Aspectos teóricos e práticos (pp.115-125). Petrópolis, RJ: Editora Vozes.

Angelini, A. L., Alves, I. C. B., Custódio, E. M., Duarte, W. F., \& Duarte, J. L. M. (1999). Manual Matrizes Progressivas Coloridas de Raven: Escala especial. São Paulo: Centro Editor de Testes e Pesquisas em Psicologia.

Biasi, F. C., \& Villemor-Amaral, A. E. (2016). Evidências de validade no Zulliger-SC para avaliação do relacionamento interpessoal de crianças. Psico, 47(1), 13-23. doi: 10.15448/1980-8623.2016.1.19990

Carvalho, A. C. M. (2015). Normatização do Teste de Zulliger SC para Crianças e Adolescentes (Dissertação de Mestrado não publicada). Programa de Pós-Graduação Stricto Sensu em Psicologia, Pontifícia Universidade Católica de Goiás, Goiânia.

Cohen, J. (1992). A power primer. Psychological Bulletin, 112(1), 155-159.

Dal'Igna, M. C. (2007). Desempenho escolar de meninos e meninas: Há diferença? Educação em Revista, 46, 241-267. doi: 10.1590/S010246982007000200010

Dornelles, P. G., \& Dal'Igna, M. C. (2015). Gênero, sexualidade e idade: Tramas heteronormativas nas práticas pedagógicas da educação física escolar. Educação e Pesquisa, 41(spe), 1585-1599. doi:10.1590/S1517-9702201508142508

Exner, J. E., \& Sendin, C. (1999). Manual de interpretação do Rorschach para o sistema compreensivo. São Paulo, SP: Casa do Psicólogo.

Faria, C. N. (2002). Puxando a sanfona e rasgando o nordeste: Relações de gênero na música popular nordestina (1950-1990). Mneme: Revista de Humanidades, 3(05). Recuperado de http://goo.gl/kBMzCV

Fensterseifer, L., \& Werlang, B. S. G. (2008). Apontamentos sobre o status científico das técnicas projetivas. Em A. E. Villemor-Amaral \& B. S. G. Werlang (Eds.). Atualizações em Métodos Projetivos para Avaliação Psicológica (pp. 15-36). São Paulo, SP: Casa do Psicólogo.

Hutz, C. S. (2015). O que é avaliação psicológica - Métodos, técnicas e testes. Em C. S. Hutz, D. S. Bandeira \& C. M. Trentini (Eds). Psicometria (pp. 11-21). Porto Alegre, RS: Artmed.

Louro, G. L. (2011). Educação e docência: Diversidade, gênero e sexualidade. Formação Docente, 03(4), 62-70. Recuperado de http:// formacaodocente.autenticaeditora.com.br/artigo/exibir/9/30/1

Miguel, F. K. (2014). Myths and facts in teaching of projective techniques. Psico-USF, 19(1), 97-106. doi: 10.1590/S1413-82712014000100010

Nascimento, R. S. G. F. (2010). Sistema compreensivo de Rorschach: Teoria, pesquisa e normas para a população brasileira. São Paulo, SP: Casa do Psicólogo.

Pacico, J. C., \& Hutz, C. S. (2015). Validade. Em C. S. Hutz, D. S. Bandeira \& C. M. Trentini (Eds). Psicometria (pp. 72-84). Porto Alegre, RS: Artmed.

Tavella, R. R., \& Villemor-Amaral, A. E. (2014). O teste de Zulliger-SC: Avaliação da criatividade em crianças. Estudos de Psicologia (Campinas), 31(4), p. 489-497. doi:10.1590/0103-166X2014000400003

Vianna, C., \& Finco, D. (2009). Meninas e meninos na educação infantil: Uma questão de gênero e poder. Cadernos Pagu, 33, 265-283. doi: $10.1590 / \mathrm{S} 0104-83332009000200010$

Villemor-Amaral, A. E., Pavan, P. M. P., Tavella, R. R., Cardoso, L. M., \& Biasi, F. C. (2016). Validity evidence of the z-test-sc for use with children. Paidéia (Ribeirão Preto), 26(64), 199-206. doi: 10.1590/1982-43272664201607

Villemor-Amaral, A. E., \& Primi, R. (2009). Teste de Zulliger no sistema compreensivo ZSC: Forma individual (1a ed.). São Paulo, SP: Casa do Psicólogo. 
Villemor-Amaral, A. E., \& Quirino, G. de S. (2013). Estudo comparativo entre indicadores afetivos das técnicas de Pfister e Zulliger. Avaliação Psicológica, 12(1), 1-7. Recuperado de https://goo.gl/200s0C

Villemor-Amaral, A. E., \& Vieira, P. G. (2016). Zulliger (CS) in Assessing the Relational Maturity of Children. Paidéia. Advance on-line publication. doi: 10.1590/1982-43272665201601

\section{Sobre as autoras}

Jamille Cavalcante de Oliveira, psicóloga graduada pela Universidade Estadual do Ceará (UECE), especialista em saúde mental, na modalidade residência, pela Universidade Federal do Ceará (UFC).

Lucila Moraes Cardoso, professora adjunta no curso de Psicologia e Programa de Pós-graduação em Educação na Universidade Estadual do Ceará e no Programa de Pós-graduação em Psicologia Universidade Federal do Ceará (UFC). 